\title{
Scaling behavior of a one-dimensional correlated disordered electronic System
}

\author{
Ibrahim Avgin \\ Department of Electrical and Electronics \\ Engineering, Ege University, \\ Bornova 35100, Izmir, Turke?*
}

(Dated: November 23, 2018)

\begin{abstract}
A one-dimensional diagonal tight binding electronic system with correlated disorder is investigated. The correlation of the random potential is exponentially decaying with distance and its correlation length diverges as the concentration of "wrong sign" approaches to 1 or 0 . The correlated random number sequence can be generated easily with a binary sequence similar to that of a one-dimensional spin glass system. The localization length (LL) and the integrated density of states (IDOS) for long chains are computed. A comparison with numerical results is made with the recently developed scaling technique results. The Coherent Potential Approximation (CPA) is also adopted to obtain scaling functions for both the LL and the IDOS. We confirmed that the scaling functions show a crossover near the band edge and establish their relation to the concentration. For concentrations near to 0 or 1 (longer correlation length case), the scaling behavior is followed only for a very limited range of the potential strengths.
\end{abstract}

*Electronic address: ibrahim@eng.ege.edu.tr 
Theoretical interest in disordered chains remains strong. Recent investigations of the correlated disordered in random or chaotic arrays revealed surprising results such as a possible breakdown of the Anderson criterion for the localization [1, 2, 3, 4, 5]. Various fields have made use of the results obtained from the study of the one-dimensional models. Random microwave transmission in a single-mode wave guide experiment [6], transport studies with GaAs-AlGaAs random dimer super lattice systems [7], and the photonic band-gap structures [8] are a few examples. Recently a new renormalization technique [9, 10] has been introduced to study the scaling behavior of the well known tight binding chain with long range correlated disorder. The authors found that the Localization Length (LL) shows scaling behavior and a cross over near the band edge. The behavior of disordered magnetic or electronic chains [11] (using proper transformations 12, 13]) can be described mathematically by a tri-diagonal tight binding model given by

$$
\left(E-\xi_{n} V\right) \psi_{n}=\psi_{n+1}+\psi_{n-1},
$$

where $E$ is energy, $V$ is the strength of the random potential with its correlated random sign $\xi_{n} . n$ is the site index.

In this paper, we study the scaling properties of a particular form of correlated disorder that is associated with spin glass chains $12,13,14,15]$. The random sign has the relation $\xi_{n}=\xi_{n-1} x_{n}$ with the following distribution $P\left(x_{n}\right)=(1-c) \delta\left(x_{n}-1\right)+c \delta\left(x_{n}+1\right)$ where the $x_{n}$ are uncorrelated between different sites and $c$ is the concentration of "wrong signs". Clearly $\xi_{n}$ is exponentially correlated, i. e., $\left\langle\xi_{n} \xi_{m}\right\rangle=(1-2 c)^{|m-n|}$ where one can define a correlation length [16] $l(c)=-1 / \ln |1-2 c|$. For $c>0.5$ the ordering is of "antiferromagnetic type" and $c=0.5$ is uncorrelated case since the correlation length is zero. As seen in Fig. 1, for $c$ approaching 0 or 1 the correlation length diverges.

Previous studies of the disordered chain problem showed that the electronic wave function decays exponentially with a distance 17] characterized by the real part of the Lyapunov exponent 18] whose imaginary part is also related to the Integrated Density of States (IDOS) 18]. In our work numerical calculations are carried out for chains of $10^{8}$ sites using the negative eigenvalue counting technique introduced by Dean [19] providing direct computation of the Lyapunov exponent.

Recently the scaling properties arising from long range correlated disorder [9, 10] were investigated using the renormalization approach. The authors have argued that their analyses 
work close to the band edge where the characteristic wavelength diverges since neighboring lattice sites move as blocks. Here we adopt their results for the correlated case discussed above.

The complex Laypunov exponent [18] is defined as $\Gamma(E, V, c)=\lim _{N \rightarrow \infty} \frac{1}{N} \ln \frac{\psi_{N}}{\psi_{0}}$. The space decimation procedure [10] leads to redefining the random potential by its mean value over the block, i.e., $V_{N}=\sum_{n=1}^{N} V \xi_{n}$. The square of this scaled potential has finite value proportional to $N$ with increasing $N$ for all concentrations as displayed in Fig. 1. For $c>0.5$, the summation has alternating values particularly persistent for $c \rightarrow 1$ but eventually reaches to a limiting value discussed below for larger $N$ (see Fig. 1). For uncorrelated case exact scaling results exist for the $L L$ and the IDOS defined via the Lyapunov exponent. Near the band edges $(E \rightarrow 2$ and $V \rightarrow 0$ ) the Lyapunov exponent displays a scaling law of the form 18] $\Gamma \approx<V^{2}>^{1 / 3} h\left(\frac{2-E}{<V^{2}>^{2 / 3}}\right)$ where $h$ explicitly known scaling function. For the correlated case, it was assumed that the Lyapunov exponent 10] has similar power-law relation to the second moment of the redefined random potential (note that the first moment is zero) $<V_{N}^{2}>$ which can be written for our case as:

$$
<V_{N}^{2}>=\frac{V_{N}^{2}}{N}=\frac{1-c}{c} V^{2} .
$$

Detailed account of this factor is given in Ref. 12, 20] This factor (in fact its $1 / 3$ power) $\frac{1-c}{c}$ is shown in Fig. 1 and it plays a role in the scaling behavior discussed below. Unlike the correlation length it diverges as $c \rightarrow 0$ while it remains finite as $c \rightarrow 1$.

At the band edge $E=2$, according to the analysis of Ref.[10] the space decimation for blocks of b sites results in the following scaling: the strength of the potential must hold (to preserve the form of the Eq. (II) $V \rightarrow V_{b}=b V$ (see Eq.(7-11) of Ref.[10]), hence the second moment transforms $\left\langle V_{N_{b}}^{2}\right\rangle=b \frac{1-c}{c}(b V)^{2}=b^{3}\left\langle V_{N}^{2}\right\rangle$. This corresponds to their 10] case of $\gamma=1$. Thus The Lyapunov exponent at the band edge can be written in a form 10, 18] $\Gamma(E=2, V, c)=\left(<V_{N}^{2}>\right)^{1 / 3}=\left(V^{2} \frac{1-c}{c}\right)^{1 / 3}$. The power of the Lyapunov exponent is the same as the uncorrelated case [18] but the coefficient is different. The factor involving concentration was recovered before using phenomelogical arguments in the context of the spin glass chain [12, 20]. The exact results for uncorrelated case $c=0.5$ are well known [18] given by $I D O S=-\frac{1}{\pi} \Im \Gamma=0.159 V^{2 / 3}$ and $\frac{1}{L L}=\Re \Gamma=0.289 V^{2 / 3}$. In an earlier work we showed that the IDOS and the LL [12, 14, 20] results for various concentrations scaled with a similar factor where data collapsed well to the exact calculation at $c=0.5$. 

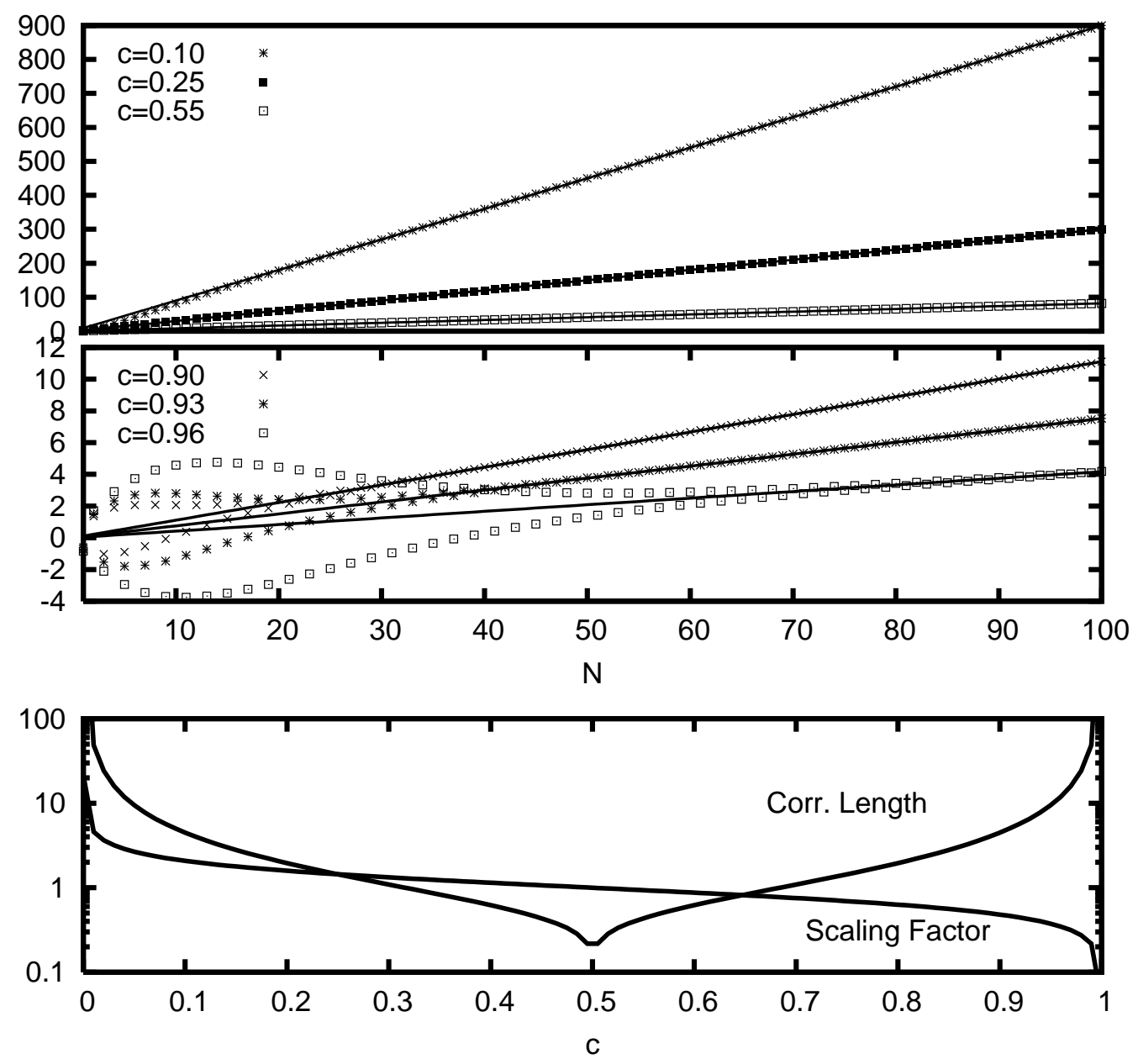

FIG. 1: The lower panel is a plot of the scaling factor $\left(\frac{1-c}{c}\right)^{1 / 3}$ and the correlation length $l(c)$ versus c. $l(c)$ is symmetric about $c=0.5$ and zero at this concentration. The square of the potential $V_{N}^{2}$ versus the number of cites are presented at the upper panels with respective concentrations. The lines are $V_{N}^{2}=\frac{1-c}{c} N$ obtained for a large $N$ and the points are calculated by a finite summation of $V_{N}^{2}$.

For uncorrelated case it is known that there is a cross over limit when $w \equiv 2-E$ is positive and very small [9, 18]. In this limit, the Lyapunov exponent can be written in the form

$$
\Gamma(w, V, c) \sim\left(V^{2} \frac{1-c}{c}\right)^{1 / 3} F(X)
$$

where $X \equiv w\left(V^{2} \frac{1-c}{c}\right)^{-2 / 3}$ and $F(X)$ is the scaling function. From the previous studies it was found [9, 18] that the scaling function has different behaviors for $X<<1$ and $X>>1$, 
hence there is a cross over between these two limits. It was argued [9] that in this system the cross over is a consequence of the competition between the characteristic length $\lambda$ of the wave function and the LL and the two asymptotic regimes can be obtained by the dominance of the LL or $\lambda$. When $\lambda>>L L$ where $w \rightarrow 0$ the wave function is independent of $\lambda$ since it would decay over a distance of $\lambda$ and the LL does not deviate much from its value at the band edge so that $F(x) \rightarrow$ constant. In the other limit $L L>>\lambda$ where the wave function would have considerable oscillations before decaying, the LL has a different functional dependence on the strength of the random potential [9, 18], i. e., $L L \sim<V^{2}>$ since $w$ dominates the dynamics yielding $F(X) \rightarrow X$.

Insight can be gained if we adopt the Coherent Potential Approximation (CPA) results here. The CPA self energy or the coherent potential has important connections to the system's dynamics which for uncorrelated disorder [21, 22] can be written as $V_{c} \simeq\left(\frac{\left\langle V^{2}\right\rangle}{2 i}\right)^{2 / 3}$. For correlated random potentials we can substitute here the second moment of the potential given in Eq. (2) (so that the correlations between different sites are included) then the coherent potential takes this form $V_{c}=\left(\frac{1-c}{c} \frac{V^{2}}{2 i}\right)^{2 / 3}$. The dispersion relation can be obtained from the poles of the configurationally averaged $k$ dependent Green function 21] that $E-$ $\Re V_{c}=2 \cos k$ where $k$ is a wave vector. For small $k$, it can be reformulated as $k^{2} \simeq \Re V_{c}+w$. Away from the band edge the LL was calculated before [9, 18, 22] and can be expressed as $L L=\frac{8 \sin ^{2} k}{\left\langle V^{2}\right\rangle} \simeq \frac{8 k^{2}}{\left\langle V^{2}\right\rangle}$. Recalling the fact that in one dimension $k$ is proportional to the IDOS, both the LL and the IDOS can be rearranged yielding the scaling functions respectively $F(x)=L L\left(\frac{1-c}{c} V^{2}\right)^{1 / 3}=8\left(2^{-5 / 3}+X\right)$ and $G(X)=I D O S\left(\frac{1-c}{c} V^{2}\right)^{-1 / 3}=\pi^{-1} \sqrt{2^{-5 / 3}+X}$ where $G(X)$ has different asymptotic behavior when $X>>1, G(X) \rightarrow \sqrt{X}$.

We present our numerical and the CPA results below. We have calculated the scaling functions $F(X)$ and $G(X)$ for chains of $10^{8}$ sites using the negative eigenvalue counting technique. As shown in Fig. 1 (semi-logarithmic plot) the correlation length goes to zero as $c \rightarrow 0.5$ and increases with increasing $|c-0.5|$ with a rapid increase developing near $c \rightarrow 1,0$. The results presented in Fig. 2 are for shorter correlation lengths where $c=$ $0.1,0.25,0.5,0.75,0.9$ and for $w=10^{-4}$ and $10^{-3}$. The data clearly indicate that there is a scaling function for both the LL and IDOS. The CPA under estimates the scaling function for the LL but reproduces the IDOS results rather well as shown in figures presented in this section. We have found that for $w>10^{-4}$ the scaling functions some what deviate from the expected scaling behavior near the lower values of $X$. However for $w<10^{-4}$ they follow the 


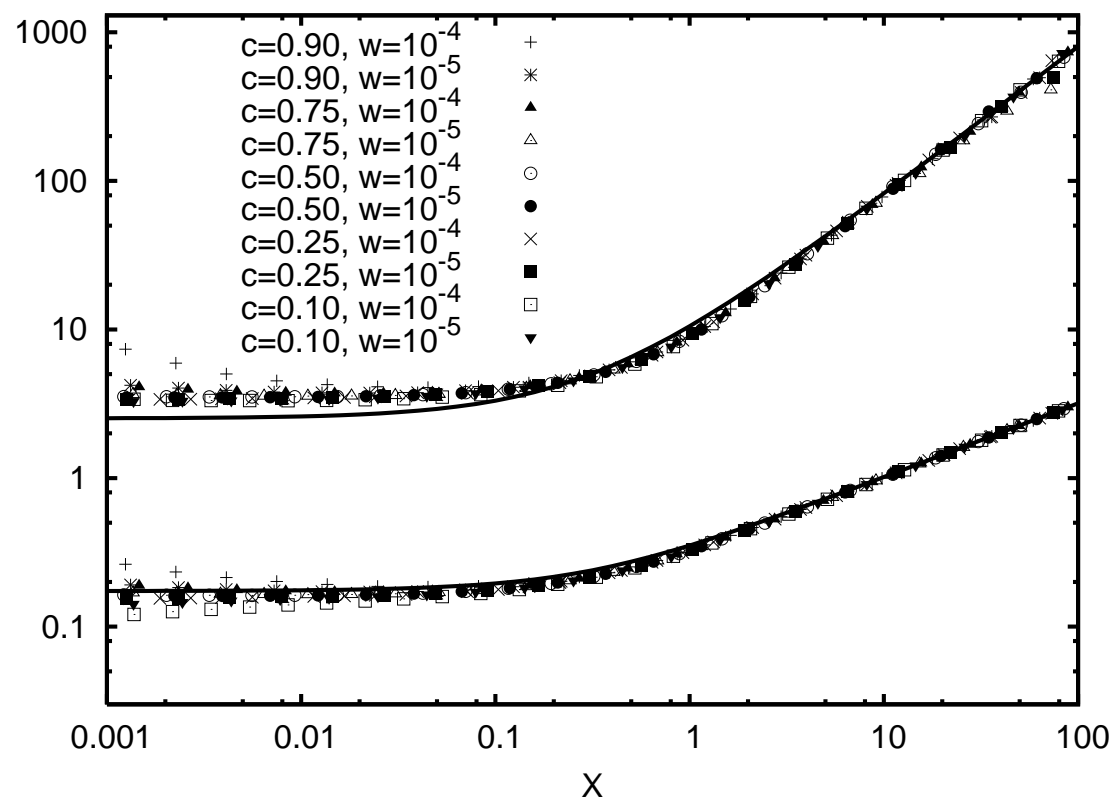

FIG. 2: plot of $F(X)$ (top) and $G(X)$ (bottom) as function of the scaling variable $X \equiv$ $w\left(V^{2} \frac{1-c}{c}\right)^{-2 / 3}$ strength $V$. The lines are the CPA results. The data displayed for the concentrations $c=0.1,0.25,0.5,0.75,0.9$ and the two energies $w=2-E=10^{-4}$ and $10^{-5}$.

predicted results.

For concentrations near 0,1 a region of rapidly increasing correlation length, we produced two sets of data displayed in Fig. 3 and Fig. 4. In Fig. 3 the scaling functions are displayed for concentrations $c=0.1,0.05,0.9$, and 0.95 , where the rapid increase in the correlation length starts. The scaling behavior is observed for only $w \geq 10^{-6}$. Note that we obtained similar, even better scaling behavior for the concentrations presented in Fig. 2 with $w \geq 10^{-6}$ values.

The scaling behavior is also clearly revealed in Fig. 4 where the deviations near the lower limit are generally largest for $w=10^{-6}$. The concentrations here are very close to 0,1 , as presented in figure caption, so one has to go to very small values of $w$ (fixed $V$ ) to see the scaling behavior. The shrinking of the scaling region in $w$ as $c \rightarrow 0,1$ is consistent with the behavior for $c=0,1$, where the correlation length is infinite. The collapse of the data for different values of $c$ is a feature of the model that reflects the fact that both the second moment (Eq. (2) and the correlation length are functions of $c$. The IDOS results also deviate for this range of concentrations. Thus as the correlation length is increased, the scaling behavior can be observed only for smaller and smaller values of $w$; however, we 


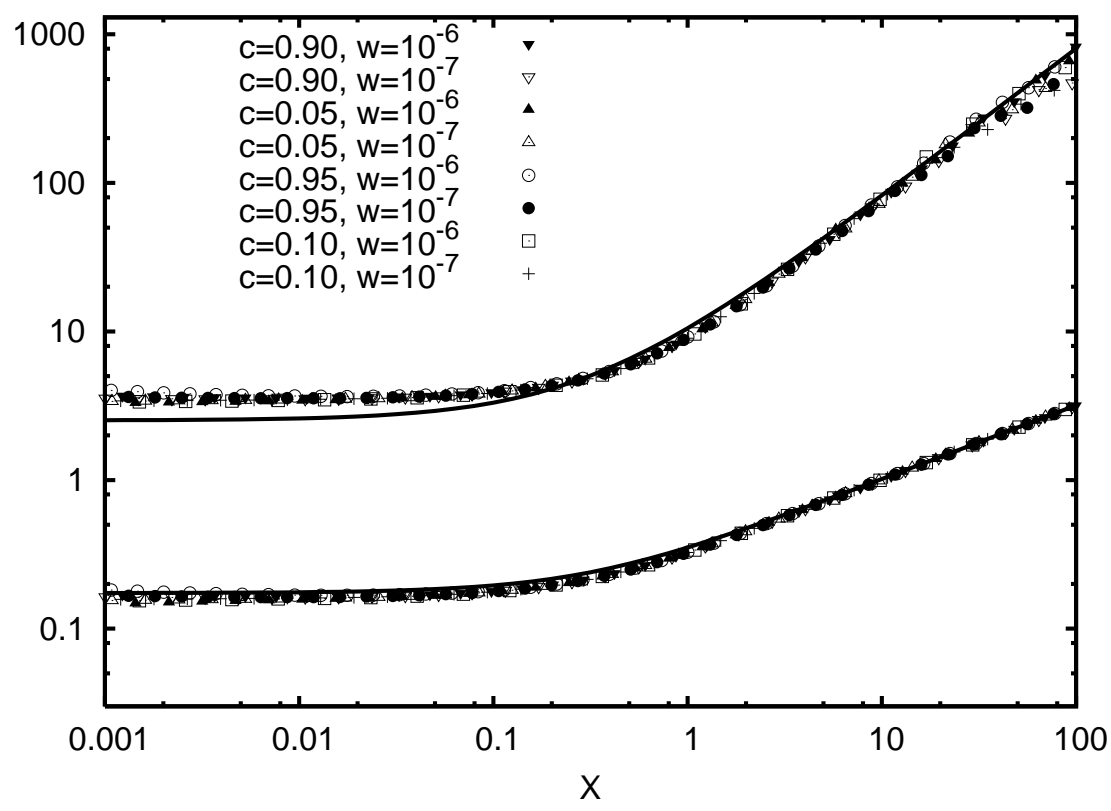

FIG. 3: plot of $F(X)$ (top) and $G(X)$ (bottom) as function of the scaling variable $X \equiv$ $w\left(V^{2} \frac{1-c}{c}\right)^{-2 / 3}$ strength $V$. The lines are the CPA results. The data displayed for the concentrations $c=0.1,0.05,0.9,0.95$ and the two energies $w=2-E=10^{-6}$ and $10^{-7}$.

found that $w$ cannot be reduced as much as desired. Hence after certain small $w$, deviations starts this time particularly at higher $X$ sides. Thus there is a trade-off in building the scaling variable $X$ since it depends on the three parameters $w, c$ and $V$ and only delicate combination of these parameters produces scaling and the crossover behavior. Stronger deviations presented in Fig. 3-4 at very small $X$ for the concentrations near 0,1 show this sensitive dependence. Because very small concentrations, the scaling factor becomes very small or very large, therefore the Lyapunov exponent must be computed in a region of a very small or a very large potential strengths at very small $w$ wherein the computational errors inevitable or the scaling behavior of the Lyapunov exponent holds only very limited potential strengths $V$.

We have studied binary correlated disordered chains both analytically and numerically. The LL and the IDOS are computed for various concentrations. We found that they both showed a scaling behavior and a crossover. The behavior of the scaling functions predicted by the renormalization group techniques [9, 10] is observed. The data calculated numerically for various concentrations with increasing correlation length largely collapsed to scaling functions belonging to the LL and IDOS separately. The scaling factor as a function of 


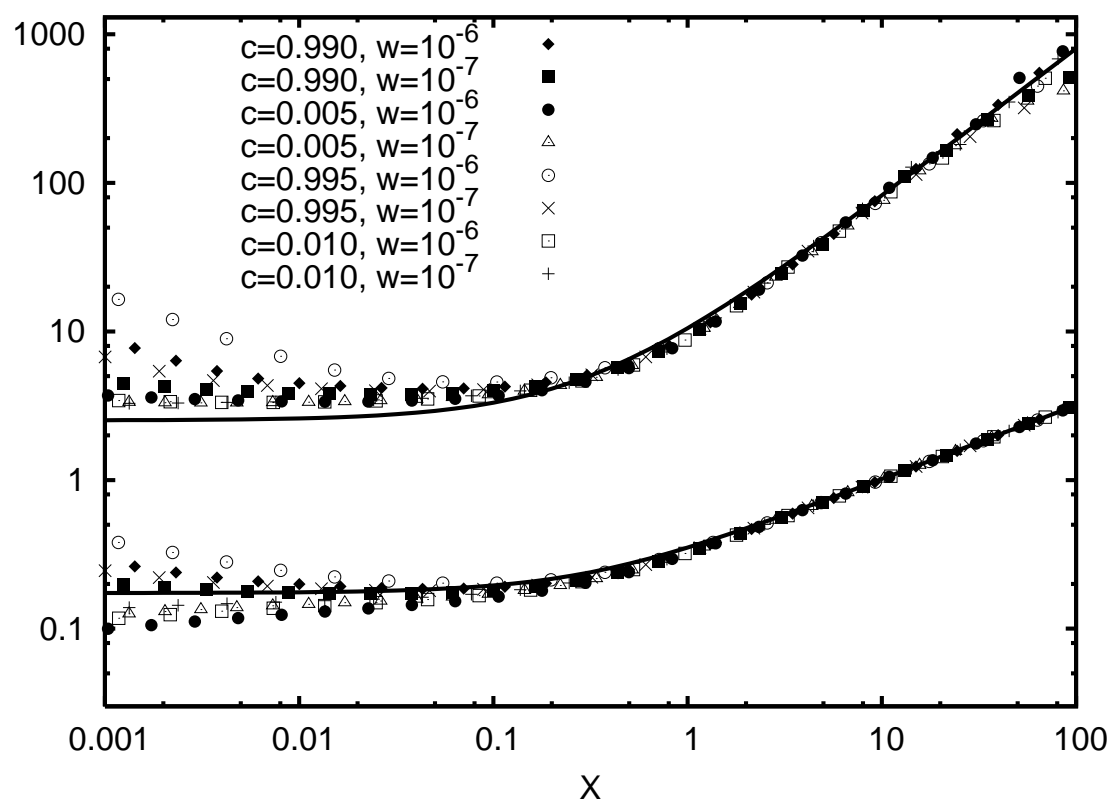

FIG. 4: plot of $F(X)$ (top) and $G(X)$ (bottom) as function of the scaling variable $X \equiv$ $w\left(V^{2} \frac{1-c}{c}\right)^{-2 / 3}$ strength $V$. The lines are the CPA results. The data displayed for the concentrations $c=0.01,0.005,0.99,0.995$ and the two energies $w=2-E=10^{-6}$ and $10^{-7}$.

concentration plays a key role in the scaling behavior of the LL and IDOS data. The scaling behavior obtained here holds for a more limited range of $w$ values than that obtained by Russ et al. 9]. For instance we did not see any scaling behavior when $w>10^{-4}$, whereas they presented the scaling behavior for $w=10^{-1}-10^{-5}$. For the scaling near the limiting concentrations, some scattered data are obtained for lower values of the scaling variable $X$. We have used scaling arguments and established the scaling functions for both the LL and the IDOS using the CPA results. The CPA results reproduced data for the ILL rather qualitatively but surprisingly for the IDOS it reproduced the data rather well. This study revealed that if the second moment of the correlated random potential is calculated, then the CPA results can be implemented as the way presented here even though the CPA was developed mainly for noncorrelated random potentials.

We have benefited from discussions with Prof. D. L. Huber. This work is partially sponsored by the Scientific end Technical Research Council of Turkey (TUBITAK).

[1] H. Yamada, Phys. Rev. B 69, 14205(2004). 
[2] R. P. A. Lima and M. L. Lyra, Phys. Rev. B 65, 104416 (2002).

[3] J. V. Kantelhardt, S. Russ, A. Bunde, S. Havlin and I Webman, Phys. Rev. Lett. 84, 198 (2000) and F. A. B. F. de Moura and M. L. Lyra, Phys. Rev.Lett.81, 3735 (1998).

[4] L. Tessieri, J. Phys. A 35, 9585 (2002) and F. M. Izrailev and A. A. Krokhin, Phys. Rev. Lett.82, 4062(1999).

[5] L. I. Deyc, M. V. Erementchouk, and A. A. Lyansky, Phys. Rev. B 67, 24205(2003).

[6] U. Kuhl, H. J. Stöckmann Physica 9 E 384 (2001).

[7] F. Kuckar, H. Heinrich and G. Bauer, Localization and confinement of electrons in semiconductors (Springer-Verlac,Berlin,1997)

[8] M. Bayindir, S. Tariseven and E. Ozbay Appl. Phys. A 72117 (2001).

[9] S. Russ, Phys. Rev. B 66, 12204(2002).

[10] S. Russ, S. Havlin and I. Webman, Phil. Mag. B 77, 1449(1998).

[11] K. Ishii, Prog. Theor. Phys. Suppl. 53, 77 (1973).

[12] I. Avgin and D. L. Huber, Phys. Rev. B 48, 13625 (1993).

[13] I. R. Pimentel and R. B. Stinchcombe, Phys. Rev. B 40, 4947 (1989).

[14] I. Avgin and D. L. Huber, Phys. Rev. B 66, 12405 (2002).

[15] A. Boukahil and D. L. Huber, Phys. Rev. B 40, 4638 (1989) and Phys. Rev. B 50, 2978 (1994).

[16] A. Crisanti, G. Paladin, and G. Vulpiani, Phys. Rev. A 39, 6491 (1989).

[17] D. J. Thouless, J. Phys. C 5, 77 (1972).

[18] B. Derrida and E. Gardner, J. Phyique 45, 1283 (1984).

[19] P. Dean, Proc. Phys. Soc. (London) 73, 413 (1959).

[20] I. Avgin and D. L. Huber Phys. Rev. B 57, 8269 (1998) and I. Avgin and D. L. Huber Phys. Rev. B. 56, 2320 (1997).

[21] I. Avgin, Phys. Rev. B 59, 13554 (1999) and I. Avgin, J. Phys. Cond. Matter 8, 8379 (1996).

[22] I. Avgin, E. J. Phys. B 29, 437 (2002). 